\title{
Os efeitos da prática de atividades motoras sobre a neuroplasticidade
}

\author{
The effects of motor activities practice on neural plasticity
}

\section{Marcella de Pinho Borella' ${ }^{1}$, Tatiana Sacchelli²}

\section{RESUMO}

A neuroplasticidade dentre suas muitas definições pode ser a capacidade de adaptação do sistema nervoso, especialmente a dos neurônios, às mudanças nas condições do ambiente que ocorrem diariamente na vida dos indivíduos. Esta reorganização neural é um objetivo preliminar da recuperação neural para facilitar a recuperação da função e pode ser influenciada pela experiência, comportamento, prática de tarefas e em resposta a lesões cerebrais. Um consenso na literatura sobre a plasticidade cerebral é que o aprendizado de determinada atividade ou a somente prática da mesma, desde que não seja simples repetição de movimentos, induza mudanças plásticas e dinâmicas no sistema nervoso central (SNC). Isto porque o treinamento motor pode promover neurogênese, sinaptogênese, angiogênese, modulação pré e pós sináptica entre outros, e todos esses podem contribuir para resultados positivos na recuperação em resposta a esse treinamento. Contudo é importante precaução, pois tanto a intensidade e especificidade do tratamento, como o intervalo de tempo entre a lesão e o início da prática de atividades influenciam a recuperação da função nervosa. Portanto esta revisão quer sugerir que a neuroplasticidade parece ser dependente do aprendizado ou do treino de atividades e não simplesmente uso dependente.

Unitermos. Plasticidade Neuronal, Atividade Motora, Aprendizagem, Reabilitação, Fisioterapia.

Citação. Borella MP, Sacchelli T. Os efeitos da prática de atividades motoras sobre a neuroplasticidade

Trabalho realizado na Universidade Metodista de São Paulo, São Bernardo o Campo-SP, Brasil.

1.Fisioterapeuta, Pós-graduanda da Univesidade Meodista de São Paulo - UMESP, São Bernardo o Campo-SP, Brasil. 2.Fisioterapeuta, Mestre, Professora da UMESP, São Bernardo o Campo-SP, Brasil.

\section{SUMMARY}

The neural plasticity among its many definitions may be the ability to adjust the nervous system, especially that of neurons, to changes in environmental conditions that occur daily in the lives of individuals. This neural reorganization it is a preliminary goal of neural rehabilitation to facilitate the function and can be influenced by the experience, behavior, practice of tasks and in response to cerebral lesions. A consensus in the literature on brain plasticity is that the learning of a given activity or only practices the same, provided it is not simple repetition of movements, lead plastic and dynamic changes in the CNS. This is because the training motor can promote neurogenesis, synaptogenesis, angiogenesis, presynaptic end postsynaptic modulation among others, and all these can contribute to positive outcomes in the recovery in response to this training. But caution is important because both the intensity and specificity of the treatment, as the interval of time between injury and start of practice activity to influence the recovery of nerve function. So this review wants to suggest that the neural plasticity appears to be dependent on the learning or trainig activities and not simply dependent use.

Keywords. Neuronal Plasticity, Motor Activity, Learning, Rehabilitation, Physical Therapy.

Citation. Borella MP, Sacchelli T. The effects of motor activities practice on neural plasticity
Endereço para correspondência:

R. Elias S dos Anjos, 107

CEP 09641-060, São Bernardo do Campo-SP, Brasil. e-mail: marcella_borella@yahoo.com.br

Artigo de Revisão

Recebido em: 03/03/2008

Revisado em: 04/03/2008 a 14/05/2008

Aceito em: 15/05/2008

Conflito de interesses: não 


\section{INTRODUÇÃO}

Por definição a neuroplasticidade é qualquer modificação do sistema nervoso que não seja periódica e que tenha duração maior que poucos segundos ${ }^{1}$. Ou ainda a capacidade de adaptação do sistema nervoso, especialmente a dos neurônios, às mudanças nas condições do ambiente que ocorrem no dia a dia da vida dos indivíduos, um conceito amplo que se estende desde a resposta a lesões traumáticas destrutivas até as sutis alterações resultantes dos processos de aprendizagem e memória ${ }^{2}$.

A plasticidade neural é maior durante a infância, e declina gradativamente, sem se extinguir na vida adulta, e ocorre tanto no hemisfério intacto como no lesionado ${ }^{3}$. Há várias formas de plasticidade, regenerativa, axônica, sináptica, dendrítica, somática e habituação que é uma de suas formas mais simples ${ }^{2}$.

A neuroplasticidade pode ter valor compensatório, mas nem sempre isso ocorre, porque as transformações neuronais que respondem ao ambiente nem sempre restauram funções perdidas. Ao contrário às vezes produzem funções mal adaptativas ou patológicas ${ }^{2}$.

Por volta de 1800 a hipótese da neuroplasticidade começou a ser descrita quando estudos sugeriram que porções sobreviventes do cérebro alteravam sua atividade funcional de modo a fazer às vezes de outra para contribuir com sua recuperação ${ }^{4}$. Mas foi somente em 1906 que o termo plasticidade pode ter sido introduzido por Ernesto Lugano um psiquiatra italiano ${ }^{5}$.

Uma visão mais moderna dessa hipótese foi aprimorada em 1948 e dizia: "A aplicação de um estímulo gera dois níveis de mudanças no sistema nervoso. A primeira é a excitabilidade e a segunda são transformações funcionais permanentes que ocorrem em sistemas particulares de neurônios, devido à aplicação de estímulos apropriados é o que chamaremos de plasticidade neural e as mudanças correspondentes de mudanças plásticas"6.

Já na década de 60 alguns pesquisadores fizeram grandes descobertas postulando que conexões neurais do córtex são intensificadas e remodeladas por nossas experiências ${ }^{7,8}$.

Nos anos 80 a percepção dos cientistas sobre a capacidade plástica regenerativa do sistema nervoso central (SNC) de mamíferos adultos começou a mudar, com os experimentos de Albert Aguayo que utilizou ratos adultos submetidos à transecção do nervo óptico. Neste estudo Aguayo tirou duas importantes conclusões. A primeira é que os axônios centrais são capazes de regenerar, desde que estejam em contato com o microambiente do sistema nervoso periférico (SNP). E a segunda o microambiente do SNC não favorece o crescimento regenerativo dos axônios centrais ${ }^{2}$.

Mas foi somente nas duas últimas décadas que vários relatos de plasticidade tem sido demonstrado em modelos experimentais em animais e em humanos, permitindo-nos a começar traçar mecanismos implícitos. Os achados sobre neuroplasticidade tem sido observados em vários níveis de análise ${ }^{4}$.

Os trabalhos atuais relatam que a reorganização neural guiada de uma maneira que facilite a recuperação da função é um objetivo preliminar da recuperação neural ${ }^{4}$. Os estudos com humanos confirmam que essa reorganização pode ser facilitada incorporando treinamento repetitivo ${ }^{9,10}$, prática de tarefas específicas ${ }^{11}$, treinamento sensorial ${ }^{12}$ e prática mental $^{13}$, todas integradas as estratégias de reabilitação ${ }^{14}$.

Em todas as doenças neurológicas um programa de tratamento que incorpore principalmente o treino de atividades funcionais, é sempre essencial para uma maior independência dos pacientes, acredita-se que um dos elementos que permitem a evolução clínica desses pacientes é que o treino dessas atividades interfira de forma benéfica na neuroplasticidade estimulando-a. Porém essa hipótese e os fatores determinantes da plasticidade neural após uma lesão não estão totalmente esclarecidos, então a intenção desta revisão é atualizar nossos conhecimentos sobre a relação entre a prática de atividades motoras ou a aprendizagem das mesmas com a neuroplasticidade.

\section{MÉTODO}

Para compor este trabalho, utilizou-se de revisão bibliográfica de artigos científicos publicados nos anos de 1993 a 2007 e pesquisados nas bases de dados MEDLINE, LILACS e SCIELO através das palavras-chave plasticity, neural plasticity, rehabilitation, physiotherapy, physical therapy, motor function, motor activity, motor task, motor learning, task-specific, activity-dependent e também capítulos de livros referentes a neurociências. Todos os artigos que tratavam da relação entre neuroplasticidade e atividade motora, sendo estes de revisão da literatura ou pesquisas com animais ou seres humanos foram selecionados. 


\section{RESULTADO}

Foram encontrados 110 artigos, todos na língua inglesa, 95 correspondiam especificamente ao tema pretendido e foram utilizados para compor esta revisão.

\section{Mecanismos de plasticidade cerebral}

Há amplas evidências sugerindo que depois de uma lesão, alterações plásticas acontecem no cérebro para compensar a perda da função nas áreas prejudicadas ${ }^{15,16}$.

Os mecanismos celulares são provavelmente responsáveis pela plasticidade nos humanos, porém os conhecimentos sobre esse fenômeno ainda são incompletos. Existem alguns mecanismos que estão sendo estudados, o primeiro é que as alterações no equilíbrio entre excitação e inibição podem acontecer muito rapidamente. Este processo depende da região de conexões anatômicas de neurônios e vias neurais no território influenciado pelo treino das funções. Algumas zonas podem servir como obstáculos para a inibição tônica. Se a inibição é removida, a região da influência pode rapidamente aumentar ou ser desmascarada; no processo chamado de desmascaramento. $\mathrm{O}$ segundo processo é a intensificação ou diminuição das sinapses existentes, no processo semelhante à potenciação a longo prazo (LTP) ou depressão a longo prazo (LTD) ${ }^{17,18}$. O terceiro processo é a alteração na excitabilidade da membrana do neurônio ${ }^{19}$. O quarto e último são as alterações anatômicas que incluem o brotamento de novos terminais axônicos e formação de novas sinapses (sinaptogênese). Esses processos ocorrem em diferentes períodos de tempo e nem sempre mutuamente ${ }^{20}$.

A plasticidade acontece não somente no córtex, mas pode acontecer também em regiões subcorticais como o tálamo e tronco cerebral ${ }^{21,22}$.

\section{Reorganização cortical}

No encéfalo adulto, as áreas corticais constantemente ajustam o modo como processam informação, conservando a capacidade de desenvolver novas funções. Os mapas das áreas funcionais do córtex cerebral são produzidos pelo registro da atividade neural, em resposta à estimulação sensorial ou durante as contrações musculares ativas ${ }^{1}$.

Diferentes partes do corpo são representadas em diferentes locais do córtex motor primário. Assim como a representação dos movimentos indivi- duais é amplamente distribuída e sobreposta nesse córtex. Sugere-se que a representação dos braços, mãos e dedos e também dos músculos são múltiplas e sobrepõem-se ${ }^{23}$.

As áreas de representação cortical "mapas corticais" podem ser modificadas pela experiência, treinamento e em resposta a lesões cerebrais ${ }^{23-25}$. A plasticidade sináptica e o número de espinhas dendríticas nas conexões horizontais do córtex são provavelmente explicadas pela reorganização desses mapas $^{24,25}$. Essas representações corticais também podem ser alteradas por várias manipulações, incluindo alterações em inputs aferentes sensoriais e estimulação repetitiva do córtex ${ }^{23}$. Em um estudo, pesquisadores amputaram o dedo de um macaco e constataram que o lugar no córtex motor antes ativado por aquele dedo logo foi invadido por projeções de neurônios transmitindo informações de um dedo adjacente, indicando que a região cerebral originalmente dedicada ao dedo perdido agora monitorava e processava informação de um dedo adjacente, comprovando que os mapas neurais se moviam $^{8}$.

A reorganização cortical também é considerada uma base potencial para a recuperação nos estágios precoces e tardios de doenças neurológicas ${ }^{23-29}$. A performance comportamental dos animais ${ }^{23,30}$ e também do tipo de treinamento que receberão durante a recuperação influenciarão diretamente nesta reorganização ${ }^{31,32}$.

\section{Efeitos da reabilitação sobre a plasticidade}

Um consenso na literatura sobre a plasticidade cerebral é que a prática de tarefas motoras induz mudanças plásticas e dinâmicas no $\mathrm{SNC}^{33}$.

As conexões neurais corticais podem ser remodeladas pelas nossas experiências ${ }^{3,34}$ e também durante o aprendizado ${ }^{7-27,32,35,36}$. A prática de atividade motora e a aprendizagem de habilidades podem alterar sinapses ou reduzir eventos moleculares na área perilesionada ou nas áreas mais remotas do córtex, incluindo as não diretamente prejudicadas ${ }^{37-40}$.

Os experimentos envolvendo animais lesados ou não e a reabilitação mostram que os mapas de representação cortical são alterados, sinapses alteram sua morfologia, dendritos crescem, axônios mudam sua trajetória, vários neurotransmissores são modulados, sinapses são potencializadas ou deprimidas $^{3}$, novos neurônios diferenciam-se e sobrevivem, ocorre aumento da mielinização dos neurônios remanescentes e maior recrutamento de pools de 
motoneurônios, transferindo a função das áreas prejudicadas para as áreas adjacentes preservadas ou correlatas $^{32-45}$. Com poucas exceções, estas investigações não têm determinado quais aspectos da neuroplasticidade estão associados com eventos adaptativos ou mal adaptativos ${ }^{46}$.

\section{A importância das experiências individuais}

O comportamento e as experiências individuais são capazes de levar a alterações plásticas em cérebros adultos normais ou lesionados ${ }^{23-31,47-50}$, essas alterações podem acontecer em múltiplos níveis do $\mathrm{SNC}^{16}$. A plasticidade experiência-dependente é acompanhada pelo aumento do número de sinap$\operatorname{ses}^{7}$ e no pós lesão essa experiência é o maior modulador das alterações neurofisiológicas e neuroanatômicas nos tecidos não lesados ${ }^{23}$.

A pré-prática da experiência acelera a prática relacionada com as alterações da ativação funcional no cérebro. Isso sugere que a recuperação do cérebro prejudicado é melhor se o indivíduo tiver melhor educação e maior atividade intelectual antes da lesão ${ }^{51}$.

Como já mencionado anteriormente há uma relação entre experiência e alteração cortical, esta pode ser observada em músicos que tocam instrumentos de cordas, pois a região do córtex que controla a mão que dedilha é maior que a região destinada à outra mão, e os dedos mais usados ocupam mais espaço. E também em leitores de Braile por ocorrer expansão da representação dos dedos e ativação do córtex visual quando tocam as saliências $20-23,52-54$.

\section{Plasticidade atividade-dependente}

A capacidade que a prática de atividades motoras possui para influenciar uma lesão cerebral é complexa devido à dinâmica neurocelular e alterações metabólicas depois de uma lesão, que podem interferir nos efeitos dessas atividades ${ }^{55}$. Há extensa evidência que o treinamento motor pode induzir a adaptações estruturais e funcionais (plasticidade) em várias áreas motoras incluindo gânglio basal56, cerebelo $^{57}$ e núcleo rubro ${ }^{58}$.

$\mathrm{O}$ treino de tarefas ou habilidades funcionais sensibiliza árvores dendríticas que estão repletas de canais sinápticos excitáveis operando em diferentes escalas de tempo, permitindo uma sofisticada plasticidade neural ${ }^{47-60}$. Isto envolve o LTP e LTD, me- canismos existentes no SNC dos mamíferos ${ }^{2462}$. A atividade-dependente pode promover também expressão de fatores neurotróficos, neurogênese, sinaptogênese, modulação pré e pós sináptica, utilização da glicose, alteração do sistema imune e angiogêne$\mathrm{se}^{16-47,63-65}$. Porém os mecanismos moleculares ligados a atividade-dependente que modificam as conexões sinápticas e a reorganização do córtex no cérebro adulto são complexos e não totalmente entendidos ${ }^{66}$.

São conhecidas algumas substâncias como acetilcolina, dopamina, serotonina e mais recentemente o óxido nítrico ${ }^{67}$ que podem modificar a plasticidade sináptica através da prática de atividades, talvez pela facilitação do NMDA receptor ${ }^{68}$. Há evidências também que células cerebrais chamadas astrócitos atuem na plasticidade ${ }^{69}$.

Muitos estudos sugerem que a neuroplasticidade atividade-dependente é um mecanismo para a recuperação em resposta ao treinamento ${ }^{23-41,433,64,70-72}$, dependente da demanda de tarefas antes de uma simples quantidade de atividade motora ${ }^{36} . \mathrm{E}$ mais do que a repetição de movimentos é a manipulação de variáveis específicas da prática como a intensidade e especificidade da tarefa, que maximizarão o potencial da recuperação $\mathrm{o}^{33-47,73}$. Essa especificidade de treinamento induz reorganização cortical ${ }^{40}$, e o número de neurônios e a intensidade das vias neurais envolvidas na tarefa são diretamente relacionadas com a intensidade e frequência da prática da mesma ${ }^{23}$. É descrito também que um treinamento de força ou resistência isoladas, ou seja, sem estarem envolvidos em uma tarefa específica parece não induzir alterações significantes na reorganização cortical ${ }^{36,74}$ e que participações ativas (controle motor voluntário) são mais eficientes para promover alterações nas redes neurais do que movimentos passivos ${ }^{14}$.

O aumento dos mecanismos celulares e sinápticos da plasticidade promovidos pelo treino dessas atividades podem contribuir para os efeitos benéficos do enriquecimento motor, reduzirem a degeneração e promoverem a recuperação da função em cérebros lesionados ${ }^{75}$. Como pode ser observado em um trabalho no qual ratos que realizaram treino acrobático na roda, em contraste com outros que realizaram simples exercícios motores unilaterais para o membro prejudicado, demonstraram conduzir plasticidade dendrítica e sinaptogênese atividadedependente além da recuperação funcional ${ }^{45-64,76}$. Nudo também relatou que em primatas o córtex motor primário pode se reorganizar depois da lesão se uma habilidade motora for retreinada ${ }^{420,77}$. Estes 
resultados sugerem que a reabilitação pelo treino de habilidades depois de uma lesão é necessária para prevenir a piora do quadro clínico ${ }^{78}$.

Os efeitos do treinamento de tarefas motoras sobre a neuroplasticidade também foi encontrado em pesquisas com seres humanos, como em um estudo com 10 pacientes com acidente vascular cerebral (AVC) e paresia em membro superior que receberam 4 semanas de sessões diárias de tratamento com tarefas funcionais e obtiveram melhora nas atividades como rolar na cama, tomar banho, lavar louça, abotoar, entre outras e ativação do córtex primário sensoriomotor, córtex pré motor ipsilateral e contralateral e cerebelo ipisilateral ${ }^{30}$. Também em um estudo semelhante só que com 8 semanas de tratamento encontrou-se aumento de $20 \%$ na melhora da independência funcional, incluindo tarefas motoras finas, discriminação sensorial e performance musculoesquelética, foi observado ainda que a prática de exercícios em casa maximizou a recuperação e os ganhos foram mantidos por 3 meses $^{14}$. Langhammer e Stanghelle em seu trabalho também obtiveram resultados positivos na recuperação funcional de 61 pacientes que foram tratados por três meses $^{79}$. Então sessões de reabilitação com rotina diária de exercícios para o membro afetado levam a redução de degeneração secundária e melhora dos resultados do comportamento motor ${ }^{75}$.

Como já descrito neste e em vários artigos é conhecido que a prática de habilidades motoras estimula a remodelagem cortical. Os resultados do estudo de Friel $^{80}$ indicou que a adicional perda da representação da mão acontece a não ser que animais recebam treinamento de habilidades na mão prejudicada $^{31}$. Um grupo de pacientes que recebeu fisioterapia por um período entre 4 e 8 semanas por uma hora e meia demonstrou que a atividade-dependente aumentou as áreas de representação corticais e melhorou a função motora ${ }^{23,81}$.

Experiências com gatos adultos depois de lesão medular que receberam treinamento específico do andar, obtiveram como resultado melhora na performance do caminhar ${ }^{36-83}$. Pacientes que sofreram lesão C7 e C8 tiveram expansão da representação da área do braço na direção da representação da mão depois do tratamento ${ }^{27}$. Portanto ambos estudos sugerem que há algum grau de plasticidade atividade-dependente na medula espinhal e mais especificamente nos mecanismos do controle locomotor $^{3683}$. Durante a reorganização dos circuitos medulares depois da lesão, o que determinará se haverá a dependência em membros superiores ou inferiores, é a frequência dos modelos de atividades nos circuitos sensoriomotores experiência-específica. Por exemplo, a repetição de uma performance de uma tarefa motora, semelhante a subir um degrau, por um período de semanas aumenta a probabilidade de completo sucesso na tarefa ${ }^{36,83}$. A quantidade de treinamento motor induz alterações biomecânicas e eletrofisiológicas na medula espinhal que são associadas com melhora da performance motora depois da lesão ${ }^{36,83}$.

\section{Plasticidade aprendizagem-dependente}

O aprendizado depende de alterações persistentes e da longa duração da força das conexões sinápticas. Com a repetição de tarefas, ocorre redução do número de regiões ativas do encéfalo. Finalmente, quando a tarefa foi aprendida, só pequenas regiões distintas do encéfalo mostram atividade aumentada durante execução da tarefa ${ }^{1}$.

$\mathrm{O}$ aprendizado de novas habilidades esta presente para promover a plasticidade cerebral ${ }^{23-28,84,85}$. Com isso ocorre aumento dos ramos dendríticos, da densidade de espinhas dendríticas e do número de sinapses por neurônios e botões sinápticos ${ }^{23,36}$.

Não a intensidade de treinamento, mas sim o aprendizado induz a reorganização cortical ${ }^{23-28,86}$, isto pode ser observado em macacos, em ratos e gatos. Estudos recentes relatam que a sinaptogênese ${ }^{46,72}$ precede a reorganização dos mapas motores e ambas acontecem durante fases tardias do aprendizado de habilidades. Então essa reorganização e a formação de novas sinapses não contribuem para a inicial aquisição das habilidades, mas representam a consolidação das mesmas ${ }^{87}$. Essa formação sináptica que ocorre na plasticidade aprendizagem-dependente é importante para as alterações funcionais corticais ${ }^{76}$. Foram encontrados em alguns trabalhos melhora na performance motora depois dos 3 primeiros dias do treino de uma nova habilidade de alcançar, e as alterações corticais apareceram entre 7 e 10 dias desse treino $^{78}$.

Pesquisadores observaram que as diferentes ativações do córtex motor primário para a aprendizagem podem persistir por até oito semanas depois do treino de uma nova tarefa ${ }^{23}$. Durante a aprendizagem a representação dos movimentos específicos é usada para o sucesso da performance das tarefas motoras, quando selecionada expande no córtex a custa de outras representações dos membros. 
Em contraste quando são realizados movimentos repetitivos que não requerem habilidade nas atividades motoras e também não evidenciam aprendizado, não produzem alterações nos mapas do córtex de ratos ou macacos ${ }^{23-36,73,77}$. Uma explicação pode ser que quando a seqüência é realizada o mapa retorna ao tamanho original refletindo diferença nos mecanismos do processo cognitivo ${ }^{23}$.

Um programa de aprendizagem motora de tarefas específicas é mais eficiente para a recuperação motora e para o nível de independência nas atividades diárias ${ }^{79}$; como comprovou um estudo com um grupo de ratos adultos que receberam cinco semanas de treinamento de uma nova tarefa de alcance comparadas com um grupo controle que não recebeu, os que foram treinados apresentaram recuperação funcional relacionada com o tamanho do córtex, enquanto que os não treinados mostraram a não reorganização dos mapas indicando falta das alterações no córtex. Assim a extensão da recuperação depois da lesão cerebral tem relação com o grau de neuroplasticidade associado com movimentos específicos $^{28}$.

Há evidências de que a aprendizagem utilizando o membro não prejudicado também possa influenciar a expressão da sinaptogênese ${ }^{45}$.

\section{Treino bilateral versus unilateral}

$\mathrm{Na}$ grande maioria dos tratamentos com pacientes neurológicos, as intervenções restringem-se a recuperação somente do membro afetado, pórem através de novos estudos como os de Bracewell ${ }^{77}$, Cauraugh et al..$^{88}$, Luft et al. ${ }^{89}$, Whitall et al. ${ }^{90}$, entre outros, observa-se que o treino bilateral, incluindo o membro não lesado, pode trazer maiores benefícios para os indivíduos do que o treino unilateral.

O planejamento e execução de movimentos bilaterais podem facilitar a plasticidade neural, e isso pode ocorrer por três mecanismos: (a) desinibição do córtex que permite maior uso das vias poupadas no hemisfério danificado; (b) aumento do recrutamento de vias ipsilaterais para suplementar as vias cruzadas danificadas do hemisfério contralateral e (c) aumento da regulação dos comandos descendentes $^{88}$.

Há evidências que alterações anatômicas são dependentes do maior uso do membro não prejudicado. Se esse membro for imobilizado durante o período de maior desenvolvimento dendrítico (0-15 dias pós lesão) a arborização dendrítica não aconte-
Alguns autores através dos resultados de seus trabalhos demonstram que o membro superior prejudicado tem uma melhor performance quando treinado em ações bimanuais do que quando utilizado sozinho. Mesmo porque a maioria das ações do dia a dia são bimanuais, há um extenso "maquinário neural" que envolve a coordenação bimanual de uma ação, por isso deve-se pensar antes de escolher o retreinamento do membro lesado ${ }^{77-90}$.

Summers et al., em um estudo com pacientes que sofreram AVC, realizarm um tratamento que envolvia tarefas funcionais como agarrar, levantar objetos, e comparou o treino bilateral com o unilateral. Os resultados encontrados foram que os indivíduos que receberam treino bilateral tiveram redução no tempo de movimento do membro lesado, aumento da habilidade funcional e diminuição do volume do mapa do músculo alvo no hemisfério não afetado comparados com os que receberam o treino unilateral. Portanto a prática bilateral pode promover melhor recuperação da função ${ }^{91}$.

O papel do hemisfério intacto na recuperação

As alterações contralaterais a lesão tem impacto na neuroplasticidade e são observadas depois de lesão unilateral no córtex de humanos ${ }^{92}$ e animais $^{55}$. Essas alterações no hemisféro intacto, como estimulação do crescimento axonal e a reinervação de regiões desnervadas no cérebro e na medula espinhal ${ }^{26}$, além da expansão dos mapas corticais ${ }^{4,41}$, podem influenciar a recuperação funcional ${ }^{2331,47} \mathrm{e}$ são responsáveis por bons resultados em pacientes neurológicos ${ }^{31-88,93}$.

Alguns estudos ${ }^{31,94}$ confirmam que o desenvolvimento das alterações morfológicas como aumento do volume dendrítico no córtex motor contralateral acontece 18 dias pós lesão e o aumento do número de sinapses por neurônios 30 dias depois da lesão.

O hemisfério contralesional parece se beneficiar também com o enriquecimento motor por habilidades específicas ou movimentos voluntários ${ }^{47,64}$. $\mathrm{O}$ prejuízo da função motora, geralmente contralateral, em algum tempo, provoca o uso compensatório do lado intacto.Em um estudo que observou 8 pacientes com boa recuperação do AVC todos tinham aumento da extensão ventral do campo da mão no córtex contralateral a lesão, tinham também grande ativação de áreas motoras suplementares, demonstrando que a plasticidade contralateral é melhor que a ipisilateral produzindo melhor recuperação ${ }^{20}$. 
Considerações importantes para uma melhor recuperação

Após lesão encefálica, tanto a intensidade do tratamento, como o intervalo de tempo entre a lesão e o início da reabilitação influenciam a recuperação da função nervosa.

É necessária precaução para começar os tratamentos, pois o uso excessivo antecipado (1-7 dias depois da lesão) no membro prejudicado causa exacerbação da lesão e piora dos resultados comportamentais $^{2346,55,95}$. Estudos que comprovam essa teoria observaram que animais submetidos a uso forçado precoce do membro apresentaram colocação errônea do mesmo, respostas diminuídas à estimulação sensorial e uso defeituoso da pata para suporte postural. Ainda mais, o córtex desses animais apresentou grande aumento da lesão e ausência de crescimento dendrítico e de brotamento ${ }^{46}$.

As diferenças individuais, como fatores genéticos, experiências pessoais em tarefas particulares, também devem ser relevantes durante o tratamento, pois interferem nos efeitos da prática. Diferentes modelos de ativação são observados em indivíduos com maior ou menor nível de familiariade com respectiva tarefa ou estímulo utilizado ${ }^{27,52}$.

\section{CONCLUSÃO}

O cérebro humano está constantemente sofrendo alterações e este é um dos motivos que dificulta o entendimento de seus mecanismos, como a regulação da neuroplasticidade após a lesão. Por isso mais pesquisas, principalmente com seres humanos, precisam ser realizadas para uma melhor compreensão das mudanças plásticas durante a recuperação das funções nervosas. Mas por meio desta revisão analisando os resultados dos estudos com humanos e animais, pode-se sugerir que a prática de tarefas ou habilidades específicas, sejam elas novas ou já conhecidas, deve ser sempre o foco principal do programa de tratamento dos pacientes neurológicos, conduzindo assim a melhor recuperação funcional, pois a neuroplasticidade parece ser aprendizadodependente ou atividade-dependente e não simplesmente uso-dependente.

\section{REFERÊNCIAS BIBLIOGRÁFICAS}

1.Jacobs AB. Neuroplasticidade. In: Ekman LL. Neurociência: fundamentos da reabilitação. Rio de Janeiro: Guanabara Koogan, 2000, p.45-52.

2.Lent R. Os Neurônios se Transforman: Bases Biológicas da Neuroplasticidade. In: Lent R. Cem Bilhões de Neurônios: conceitos fundamentais de neurociências. São Paulo: Atheneu, 2004, p.134-63.

3.Johansson BB. Brain plasticity and stroke rehabilitation. Stroke 2000;31:223-30.

4.Nudo RJ. Plasticity. NeuroRX 2006;3:420-7.

5.Berlucchi G. The origin of the term plasticity in the neurosciences: Ernesto Lugaro and chemical synaptic trnsmission. J Hist Neurosci 2002;11:305-09.

6Kandel ER. As Células Nervosas e o Comportamento. In: Kandel ER, Schwartz JH, Jessel TM. Princípios da neurociência. 4rd ed. São Paulo: Manole, 2003, p.34.

7.Johansson BB. Brain plasticity in health and disease. Keio J Med 2004;53:231-46.

8.Holloway M. O cérebro reconfigurado. SCIAM 2003;17:71-7. 9.Milter W, Bauder H, Sommer M, Dettmers C, Taub E. Effects of constraint-induced movement therapy on patients with chronic motor deficits after stroke. Stroke 1999;30:586-92.

10.Wolfgang HR, Miltner W, Baudner H, Sommer M, Dettmers C, Taub E. Effects of constraint-induced movement therapy on patients with chronic motor deficits after stroke: a replication.

Stroke 1999;30:586-92.

11.Richards CL, Malouin F, Wood-Dauphinee S, Williams JI, Bouchard J, Brunet D. Task-specific physical therapy for optimization of gait recovery in acute stroke patients. Arch Phys Med Rehabil 1993;74:512-620.

12.Yekutiel M, Guttman E. A controlled trial of the retraining oh the sensory function oh the hand in stroke patients. J Neurol Neuros Psych 1993;56:241-4.

13.Page SJ, Levin P, Sisto AS, Johnson MV. Mental practice combined withy physical practice for upper limb motor deficit in subacute stroke. Phys Ther 2001;81:1455-62.

14.Byl N, Roderick J, Mohamed O, Hanny M, Kotler J, Smith A, et al. Effectiveness of sensory and motor rehabilitation of the upper limb following the principles of neuroplasticity: patients stable poststroke. Neurorehabil Neural Repair 2003;17:176-91.

15.Chollet F, Weiller C. Imaging recovery of function following brain injury. Curr Opin Neurobil 1994;4:226-30.

16.Schallert T, Leasure JL, Kolb B. Experience-associated structural events, subependymal cellular proliferative activity, and functional recovery after injury to the central nervous system. J Cereb Blood Flow Metab 2000;20:1513-28.

17. Hess G, Aizenman CD, Donoghue JP. Conditions for the induction of long-term potentiation in layer II/III horizontal connections of the rat motor cortex. J Neurophysiol 1996;75:1765-78. 18.Hess G, Donoghue JP. Long-term depression of horizontal connections in rat motor cortex. Eur J Neurosci 1996;8:658-65. 
19.Halter JA, Carp JS, Wolpaw JR. Operantly conditioned motoneuron plasticity: possible role of sodium channels. J Neurophysiol 1995;73:867-71.

20.Hallet M. Plasticity oh the human motor cortex and recovery from stroke. Brain Res Rev 2001;36:169-74.

21.Nicoletis MAL. Dynamic and distributed somatosensory representations as the substance for cortical and subcortical plasticity. Semin Neurosci 1997;9:24-33.

22.Jones EG, Pons TP. Thalamic and brain stem contributions to large-scale plasticity of primate somatosensory cortex. Science 1998;282:1121-5.

23.Nudo RJ, Plautz EJ, Frost SB. Role of adaptative plasticity in recovery of function after damage to motor cortex. Muscle Nerve 2001;24:1000-19.

24.Buonomano DV, Merzenich MM. Cortical plasticity: from synapses to maps. Annu Rev Neurosci 1998;21:149-86.

25.Hickmott PW, Merzenich MM. Local circuit properties underlying cortical reorganization. J Neurophysiol 2002;88:1288301.

26.Chen R, Cohen LG, Hallet M. Nervous System reorganization following injury. Neuroscience 2002;111:761-73.

27.Hlustík P, Mayer M. Paretic hand in stroke: from motor cortical plasticity research to rehabilitation. Cogn Behav Neurol 2006;19:34-40.

28.Ramanathan D, Conner JM, Tuszynski MH. A form of motor cortical plasticity that correlates with recovery of function after brain injury. Proc Natl Acad Sci USA 2006;103:11370-5.

29.Xerri C, Merzenich MM, Peterson BE, Jenkins W. Plasticity of primary somatosensory cortex paralleling sensoriomotor skill recovery from stroke in adult monkeys. J Neurophysiol 1998;75:2144-9.

30.Lindberg P, Schimitz C, Forssberg H, Engardt M, Borg $\mathrm{J}$. Effects of passive-active movement training on upper limb motor function and cortical activation in chronic patients with stroke: a pilot study. J Rehabil Med 2004;36:117-23.

31.Nudo RJ, Friel KM. Cortical plasticity after stroke: implications for rehabilitation. Rev Neurol (Paris) 1999;155:713-7.

32.Nudo RJ. Adaptive plasticity in motor cortex: implications for rehabilitation after brain injury. J Rehabil Med 2003;41(Suppl):7-10.

33.Fisher BE, Sullivan KJ. Activity-dependent factors affecting poststroke functional outcomes. Top Stroke Rehabil 2001;8:3144.

34.Kelly C, Foxe JJ, Garavan H. Patterns of normal human brain plasticity after practice and their implications for neurorehabilitation. Arch Phys Med Rehabil 2006;87:S20-9.

35.Kleim JA, Lussning E, Schwarz ER, Comery TA, Greenough WT. Synaptogenesis and fos expession in motor cortex of the adult rat after motor skill learning. J Neurosci 1996;16:452935 .

36.Adkins DL, Boychuk J, Remple MS, Kleim JA. Motor training induces experience-specific patterns of plasticity across motor cortex and spinal cord. J Appl Physiol 2006;101:1776-82. 37.Keyvani K, Schallert T. Plasticity associated molecular and structural events in postlesional brain. J Neuropathol Exp Neurol 2002;61:831-40.

38.Szele FG, Alexander C, Chesselet MF. Expression of molecules associated with neuronal plasticity in the striatum after aspiration and thermocoagulatory lesions oh the cerebral cortex in adult rats. J Neurosci 1995; 15:4429-48.

39.Witte OW, Stoll G. Delayed and remote effects of focal cortical infarctions: secondary damage and reactive plasticity. Adv Neurol 1997;73:207-27.
40.Nudo RJ, Milliken GW, Jenkins WM, Merzenich MM. Usedependent alterations of movement representations in primary motor cortex of adult squirrel monkeys. J Neurosci 1996;16:785807.

41.Nudo RJ, Wise B, Sifuentes F, Milliken G. Neural substrates for the effects of rehabilitative training on motor recovery after ischemic infarct. Science 1996;272:1791-4.

42.Hallet M. Plasticity in the human motor system. Neuroscientist 1999;5:324-32.

43.Jones TA, Bury SD, Adkins DL, Luke LM, Sakata JT. Importance of behavioral manipulations and measures in rat models of brain damage and brain repair. ILAR J 2003;44:144-52.

44.Schallert T, Woodlee MT, Fleming SM. Experimental focal ischemic injury: behavior-brain interactions and issues of animal handling and houding. ILAR J 2003;44:130-43.

45.Bury SD, Jones TA. Unilateral sensoriomotor cortex lesions in adult rats facilitate motor skill learning with the unaffected forelimb and training-induced dendritic structural plasticity in the motor cortex. J Neurosci 2002;22:8597-606.

46.Kozlowskj DA, James DC, Schallert T. Use dependent exaggeration of neural injury after unilateral sensorio motor cortex lesions. J Neurosci 1996;16:4776-86.

47.Biernaskie J, Corbett D. Enriched rehabilitative training promotes improved forelimb motor function and enhanced dendritic growth after focal ischemic injury. J Neurosci 2001;21:527280 .

48.Nudo RJ. Recovery after damage to motor cortical areas. Curr Opin Neurobiol 1999;6:740-7.

49.Karni A, Meyer G, Rey-Hipolito C. The acquisition of skilled motor performance: fast and slow experience-driven changes in primary motor cortex. Proc Natl Acad Sci USA 1998;95:861-8.

50.Kolb B, Whishaw IQ. Brain plasticity and behavior. Annu Rev Psychol 1998;49:43-64.

51.O' Connell MJ. Prediction of return to work following traumatic brain injury: intellectual, memory, and demographic variables. Rehabil Psychol 2000;45:212-7.

52.Hund-Georgiadis M, Cramon DY. Motor-learning related changes in piano players and non-musicians revealed by functional magnetic-resonance signals. Exp Brain Res 1999;125:417-25. 53.Munte TF, Altenmuller E, Jancke L. The musician's brain as a model of neuroplasticity. Nat Rev Neurosci 2002;3:473-8.

54.Pascual-Leone A, Wassermann EM, Sadato N, Hallet M. The role of reading activity on the modulation of motor cortical outputs to the reading hand in Braille readers. Ann Neurol 1995;38:910-5.

55.Griesbach GS, Hovda DA, Molteni R, Wu A, Gomez-Pinilla F. Voluntary exercise following traumatic brain injury: brain-derived neurotrophic factor upregulation and recovery of function. Neuroscience 2004;125:129-39.

56.Graybiel AM. The basal ganglia: learning new trics and loving it. Curr Opin Neurobiol 2005;15:638-44.

57.De Zeeuw CI, Yeo CH. Time and tide in cerebellar memory formation. Curr Opin Neurobiol 2005;15:667-74.

58.Hermer-Vazquez L, Hermer-Vazquez R, Moxon KA, Kuo $\mathrm{KH}$, Viau V, Zhan Y, et al. Distinct temporal activity patterns in the rat M1 and red nucleus during skilled versus unskilled limb movement. Behav Brain Res 2004;150:93-107.

59.Rossini PM, Altamura C, Ferreri F, Melgari JM, Tecchio F, Tombini M, et al. Neuroimaging experimental studies on brain plasticity in recovery from stroke. Eura Medicophys 2007;43:24154 . 
60.Harris KM. Structure, development, and plasticity of dendritic spines. Curr Opin Neurobiol 1999;9:343-8.

61.Luscher C, Nicoll RA, Malenka RC, Muller D. Synaptic plasticity and dynamic modulation of the postsynaptic membrane. Nat Neurosci 2000;3:545-50.

62.Kreisel SH, Hennerici MG, Bäzner H. Pathophysiology of stroke rehabilitation: the natural course of clinical recovery, usedependent plasticity and rehabilitative outcome. Cerebrovasc Dis 2007;23:243-55.

63.Cotman CW, Berchtold NC. Exercise: a behavioral intervention to enhance brain health and plasticity. Trends Neurosci 2002;25:295-301.

64.Jones TA, Chu CJ, Grande LA, Gregory AD. Motor skills training enhances lesion-induced structural plasticity in the motor cortex of adult rats. J Neurosci 1999;19:10153-63.

65.Woldag H, Hummelsheim H. Evidence-based physiotherapeutic concepts for improving arm hand function in stroke patients: a review. J Neurol 2002;249:518-28.

66.Hickmott PW, Merzenich MM. Local circuit properties underlying cortical reorganization. J Neurophysiol 2002;88:1288301.

67.Kara P, Friedlander MJ. Dynamic modulation of cerebral cortex synaptic function by nitric oxide. Prog Brain Res 1998;118:183-98.

68.Schultz W. Neural coding of basic reward terms of animal learning theory, game theory, microeconomics and behavioral ecology. Curr Opin Neurobiol 2004;14:139-47.

69.Vernadakis A. Glia-neuron intercommunications and synaptic plasticity. Prog Neurobiol 1996;6:615-21.

70.Levin HS. Neuroplasticity and brain imaging resarch: implications for rehabilitation. Arch

Phys Med Rehabil 2006;87:S1.

71.Kleim JA, Bruneau R, Calder K, Pocock D, Vandenberg $\mathrm{PM}$, MacDonald E, et al. Functional organization of adult motor cortex is dependent upon continued protein synthesis. Neuron 2003; $40: 167-76$.

72.Johansson BB. Brain plasticity and stroke rehabilitation: the willis lecture. Stroke 2000;31:223-30.

73.Plautz EJ, Milliken GW, Nudo RJ. Effects of repetitive motor training on movement representations in adult squirrel monkeys: role of use versus learning. Neurobiol Learn Mem 2000;74:27-55. 74.Kleim JA, Cooper NR, Vandenberg PM. Exercise induces angiogenesis but does not alter movement representations within rat motor cortex. Brain Res 2002;934:1-6.

75.Kleim JA, Jones TA, Schallert T. Motor enrichment and the induction of plasticity before or after brain injury. Neurochem Res 2003;28:1757-69.

76.Kleim JA, Barbay S, Cooper NR, Hogg TM, Reidel CN, Remple MS, et al. Motor learning-dependent synaptogenesis is localized to functionally reorganized motor cortex. Neurobiol Learn Mem 2002;77:63-77.

77.Bracewell RM. Stroke: neuroplasticity and recent approaches to rehabilitation. J Neurol Neurosurg Psychiatr 2003;74:1465.

78.Landers M. Treatment-induced neuroplasticity following focal injury to the motor cortex. Int J Rehabil Res 2004;27:1-5.

79.Langhammer B, Stanghelle JK. Bobath or motor relearning programme? A comparison of two different approaches of physiotherapy in stroke rehabilitation: a randomized controlled study. Neuropsychol Rehabil 2000;14:361-9.

80.Friel KM, Heddings AA, Nudo RJ. Effects of post-lesion experience on behavioral recovery and neurophysiologic reorganization after cortical injury in primates. Neurorehabil Neural Repair 2000;14:187-98.
81.Liepert J, Graef S, Uhde I, Leidner O, Weiller C. Traininginduced changes of motor cortex representations in stroke patients. Acta Neurol Scand 2000;101:321-6.

82. Carrier L, Brustein E, Rossignol S. Locomotion of the hindlimbs after neurectomy of ankle flexors in intact and spinal cats: model for the study of locomotor plasticity. J Neurophysiol 1997;77:1979-93.

83.Cai LL, Courtine G, Fong AJ, Burdick JW, Roy RR, Edgerton VR. Plasticity of functional connectivity in the adult spinal cord. Phil Trans R Soc B 2006;361:1635-46.

84.Carel C, Loubinox I, Boulanouar K, Manelfe C, Rascol O, Celsis $\mathrm{P}$, et al. Neural substrate for the effects to passive training on sensoriomotor cortcal representation: a study with functional magnetic resonance imaging in healthy subjects. J Cereb Blood Flow Metab 2000;20:478-84.

85.Zhuang P, Dang N, Waziri A, Gerloff C, Cohen LG, Hallet $\mathrm{M}$, et al. Implicit and explict learning in na auditory serial reaction time task. Acta Neurol Scand 1998;97:131-7.

86.Monfils MH, Plautz EJ, Keim JA. In search of the motor engram: motor map plasticity as a mechanism for encoding motor experience. Neuroscientist 2005;11:471-83.

87.Kleim JA, Hogg TM, VandenBerg PM, Cooper NR, Bruneau $\mathrm{R}$, Remple M. Cortical synaptogenesis and motor map reorganization occcur during late, but not early, phase of motor skill learning. J Neurosci 2004;24:628-33.

88.Cauraugh JH, Summers JJ. Neural plasticity and bilateral movements: a rehabilitation approach for chronic stroke. Prog Neurobiol 2005;75:309-20.

89.Luft AR, McCombe-Waller S, Whitall J, Forrester LW, Macko R, Sorkin JD, et al. Repetitive bilateral arm training and motor cortex activation in chronic stroke: a randomized contolled trial. JAMA 2004;292:1853-61.

90.Whitall J, McCombe-Waller S, Silver KH, Macko RF. Repetitive bilateral arm training with rhythmic auditory cueing improves motor function in chronic hemiparetic stroke. Stroke 2000;31:2390-5.

91.Summers JJ, Kagerer FA, Garry MI, Hiraga CY, Loftus A, Cauraugh JH. Bilateral and unilateral movement training on upper limb function in chronic stroke patients: a TMS study. J Neurol Scien 2007;252:76-82.

92.Chu D, Huttenlocher PR, Levin DN, Towle VL. Reorganization of the hand somatosensory cortex following perinatal unilateral brain injury. Neuropediatrics 2000;31:63-9.

93.Johansen-Berg H, Dawes H, Guy C, Smith SM, Wade DT, Matthews PM. Correlation between mtor improvements and altered $\mathrm{f}$ MRI activity after rehabilitative therapy. Brain 2002;125:2731-42.

94.Forgie ML, Gibb R, Kolb B. Unilateral lesions of the forelimb area of rat motor cortex: lack of evidence for use-dependent neural growth in the undamaged hemisphere. Brain Res 1996;710:249-59.

95.Risedal A, Zeng J, Johansson BB. Early training may exacerbate brain damage after focal brain ischemia in the rat. J Cereb Blood Flow Metab 1999;19:997-1003. 